\title{
Effects of lycopene on mouse sperm and oocyte, and in vitro fertilization outcomes
}

\author{
Patcharada Amatyakul $^{\mathrm{a}, *}$, Hathairat Kruevaisayawan ${ }^{\mathrm{b}}$, Onrawee Khongsombat ${ }^{\mathrm{c}}$, \\ Suwanthana Yamtanode ${ }^{\mathrm{a}}$ \\ a Division of Reproductive Medicine, Department of Obstetrics and Gynaecology, Faculty of Medicine, \\ Naresuan University, Muang, Phitsanulok 65000 Thailand \\ b Department of Anatomy, Faculty of Medical Science, Naresuan University, Muang, \\ Phitsanulok 65000 Thailand \\ c Department of Physiology, Faculty of Medical Science, Naresuan University, Muang, \\ Phitsanulok 65000 Thailand
}

*Corresponding author, e-mail: pamatyakul@hotmail.com

Received 6 Nov 2016

Accepted 29 Jun 2017

\begin{abstract}
Reactive oxygen species (ROS) and oxidative stress are problematic in infertile couples because of impaired sperm function and embryo development. ROS can decrease the success rate of IVF treatment. Lycopene is a carotenoid with a potent antioxidant activity. This study evaluated the antioxidative effects of lycopene on sperm and oocyte in an IVF system. Sperm motility was examined after 4-h incubation with 5, 10, and $25 \mu \mathrm{M}$ lycopene. Early and late sperm apoptosis were assessed by flow cytometry and malondialdehyde levels were measured by high performance liquid chromatography. To study the effect of lycopene on oocytes and IVF outcomes, oocytes were incubated with lycopene and then inseminated. The cleavage rate and embryo grading were assessed. The results show that lycopene had no protective effect on sperm motility. Late sperm apoptosis decreased slightly but it was not statistically significant. Outcomes of cleavage rate and good-grade embryos of oocytes incubated with lycopene had no significant differences. The higher the concentration of lycopene, the higher the oocyte degeneration rate. In conclusion, lycopene is a strong antioxidant but it may have scant antioxidative effects in an IVF system. Furthermore, oocytes incubated with high concentrations of lycopene for $1 \mathrm{~h}$ may impair the oocyte quality and cause it to degenerate. Thus supplementation of lycopene in the IVF culture media should be further studied to determine the most effective dose and incubation time.
\end{abstract}

KEYWORDS: carotenoids, oxidative stress, sperm motility, embryo developmental rate, infertility

\section{INTRODUCTION}

Infertility is becoming a big problem. Infertility is defined as the inability to conceive a pregnancy after 1 year of regular sexual intercourse and without using any method of contraception. From recent data, most of infertility causes are associated with oxidative stress which is referred to an unbalanced condition between reactive oxygen species (ROS) and antioxidants ${ }^{1-3}$. ROS or free radicals are generally formed in aerobic metabolism during the intermediate steps of oxygen reduction in mitochondria. They are superoxide anion radical $\left(\mathrm{O}_{2}{ }^{--}\right)$, hydrogen peroxide $\left(\mathrm{H}_{2} \mathrm{O}_{2}\right)$, hydroxyl radical ( $\left.\mathrm{HO}^{\circ}\right)$, and singlet oxygen $\left(\mathrm{O}^{-}\right)^{4}$. Although low levels of these ROS are needed to maintain a normal physiology, at the high levels they can be harmful to cells and tissue causing DNA fragmentation and apoptosis. Free radicals also alter the degradation rate of proteins, lipids, and nucleic acid. For example, lipid peroxides from the oxidation of phospholipids and fatty acids can initiate the lipid peroxidation in cellular membranes leading to dysfunction of membrane permeability. Recent information support the notion that free radicals are associated with many diseases such as atherosclerosis, diabetes mellitus, cancer, and Alzheimer's disease ${ }^{5,6}$.

In the reproductive system, ROS are mainly generated by sperms and leukocytes in the seminal plasma. The anatomical structure and biochemical properties of the sperm make them vulnerable to oxidative stress. The sperm plasma membrane is enriched in polyunsaturated fatty acids (PUFA) such as arachidonic acid and docosahexaenoic acid, which contain more than 2 carbon-carbon double bonds and a methylene group. The covalent bond of the methylene carbon-hydrogen bonds is weak and susceptible to abstraction. Thus hydroxyl radical can 
react at this point and initiate and propagate a lipid peroxidation reaction ${ }^{1}$. Spermatozoa have very little cytoplasmic space limited to the midpiece which contains superoxide dismutase and glutathione peroxidase. During sperm differentiation and maturation if the cytoplasmic remnants are excessive, there will be a defect on sperm function and can generate more oxidative stress ${ }^{7,8}$. PUFA at sperm membrane and small amount of ROS are however important for fusion of sperm-oocyte interaction during the process of fertilization and to control plasma membrane fluidity during the acrosome reaction ${ }^{9,10}$. ROS also have a role in sperm capacitation by stimulation of cholesterol transporter from the plasma membrane, regulation of tyrosine phosphorylation and activated cAMP at the sperm tail to induce the sperm hyperactive motility ${ }^{11}$. The target of oxidative stress in sperm is not only the plasma membrane, but also the DNA in the nucleus. It has been shown that ROS could induce cascade activity in the cytoplasm and mitochondria of the sperm midpiece and then initiate apoptosis leading to single and double DNA strand breaks and DNA fragmentation in the sperm head $^{8}$. The excessive ROS are also associated with a decrease in axonemal protein phosphorylation and a decrease in membrane fluidity which can cause sperm immobilization.

ROS have been reported to play several roles in the normal physiology of the female reproductive system. During folliculogenesis and steroidogenesis, ROS may stimulate the proliferation of theca and interstitial cells of the ovary, but high levels of ROS may inhibit this action. During oocytes maturation, ROS may have an effect on chromosome segregation and gene expression leading to oocyte maturation arrest ${ }^{12}$. Follicular fluid is a house for both ROS and antioxidants for the oocyte which may be similar to seminal plasma and sperm. The balance between ROS and antioxidants is crucial to maintain normal male and female reproductive functions. As previously mentioned, high levels of ROS occur in several infertility conditions. Pelvic endometriosis (presentation of endometrial tissue and stroma outside the uterine cavity) is a common gynaecologic disease and it is a leading cause of female infertility. This ectopic tissue can induce leukocyte and macrophage activities which are the source of ROS production. It has been shown that the peritoneal fluid collected from patients with endometriosis has high levels of lipid peroxidation and nitric oxide, which may disturb the sperm-oocyte interaction and fertilization ${ }^{12-14}$. Detrimental effects of ROS on female infertility include impaired em- bryo development leading to fragmented embryos and failed implantation causing pregnancy loss or miscarriage ${ }^{14-17}$. Nowadays, the most effective treatment of infertile couples is assisted reproductive technology (ART) and IVF. During the process of sperm and oocyte preparation, ROS can occur and fertilization rate may be interfered ${ }^{14}$. To achieve better pregnancy rate per ART cycle, ROS production should be controlled and minimized by several techniques in the ART laboratory setting. Hence antioxidants become one of the interesting issues in decreasing the oxidative stress of the IVF culture system. Two types of antioxidants are enzymatic antioxidants such as superoxide dismutase (SOD), catalase, glutathione peroxidase, and glutathione reductase and non-enzymatic antioxidants such as vitamin $\mathrm{C}$, vitamin $\mathrm{E}$, melatonin, zinc, selenium, glutathione, and carotenoids ${ }^{2,18}$.

Carotenoids or vitamin A are commonly known antioxidants because the conjugated double bonds confer the molecule antioxidative properties. Because of their lipophilicity, carotenoids may have a role to protect the cellular membranes and lipoproteins from oxidative damage ${ }^{19,20}$. Antioxidative activities of the carotenoid lycopene has been shown to be twice that of beta-carotene. Compared to $\alpha$ tocopherol (vitamin E), lycopene contains approximately 100 times more potent antioxidative effects and its antioxidative property may be the highest among carotenoids ${ }^{21}$. The tissue concentrations of lycopene in testis are higher than that in the ovary; hence lycopene may play a crucial role as an antioxidant in male reproductive system. Lycopene is also predominately found in prostate gland and evidence suggest that lycopene may decrease risk of benign prostatic hyperplasia and prostate cancer. According to recent information, lycopene has a potent antioxidative effect which may improve the sperm quality and it may be beneficial in ART setting by an increased rate of fertilization and embryo development, but data of lycopene effects on gamete and embryos are limited. Hence, this study evaluated the effect of lycopene on sperm motility, apoptosis and the IVF outcomes of oocyte treated with lycopene by using a mouse model.

\section{MATERIALS AND METHODS}

\section{Animals}

Six to eight-week-old male and female ICR mice were acclimatized at the Centre for Animal Research, Naresuan University under the controlled temperature for $22 \pm 1{ }^{\circ} \mathrm{C}$ with light:dark standard 
cycle $(12: 12 \mathrm{~h})$. All mice were fed with food and water ad libitum. The experimental protocols were approved by the Animal Ethics Committee of Naresuan University, Phitsanulok, Thailand.

\section{Preparation of lycopene}

Lycopene (Sigma-Aldrich Corp, USA) were dissolved in DMSO (Sigma-Aldrich Corp, USA) and made up to $1 \mathrm{ml}$ with distilled water preheated to $37^{\circ} \mathrm{C}$. The stock concentration was $1 \mathrm{mg} / \mathrm{ml}$. Before the experiment, lycopene was freshly prepared at various concentrations diluting with the culture media to yield lycopene $5,10,25 \mu \mathrm{M}$ and the final concentration of DMSO was less than $0.01 \% \mathrm{~W} / \mathrm{V}$ which has no effect on embryo development.

\section{Sperm preparation and evaluation for concentration and motility}

Flushing medium (FertiPro N.V., Belgium) was incubated overnight at $37^{\circ} \mathrm{C}$ with $5 \% \mathrm{CO}_{2}$ before the day of sperm collection. Five male mice were sacrificed and the epididymis was dissected and placed into $700 \mu \mathrm{l}$ of flushing medium under oil (Life Global group, USA). The sperm samples collected from 5 males were pooled together and examined for the concentration and motility by loading $10 \mu \mathrm{l}$ of sample on the Makler counting chamber (Sefimedical instruments LTD). The motility parameters were measured as motile and non-motile sperm. The pooled sperm sample was divided into 8 groups as follows: (1) control group with no treatment; (2) control group with hydrogen peroxide $10 \mu \mathrm{M}$; (3) treatment group with lycopene $5 \mu \mathrm{M}$; (4) treatment group with lycopene $10 \mu \mathrm{M}$; (5) treatment group with lycopene $25 \mu \mathrm{M}$; (6) treatment group with lycopene $5 \mu \mathrm{M}$ and hydrogen peroxide $5 \mu \mathrm{M}$; (7) treatment group with lycopene $10 \mu \mathrm{M}$ and hydrogen peroxide $5 \mu \mathrm{M}$; and (8) treatment group with lycopene $25 \mu \mathrm{M}$ and hydrogen peroxide $5 \mu \mathrm{M}$.

The sperm in all groups was incubated for sperm capacitation in a $5 \% \mathrm{CO}_{2}$ incubator, $37^{\circ} \mathrm{C}$ for $4 \mathrm{~h}$ and then reevaluated for sperm motility. The sperm with a concentration of $10^{6}$ cells $/ \mathrm{ml}$ were immediately prepared for evaluation of sperm apoptosis by flow cytometry; $200 \mu \mathrm{l}$ of the specimen from each group was collected for detection of malondialdehyde (MDA) levels.

\section{Detection of sperm apoptosis by flow cytometry}

Sperm were washed twice with $\mathrm{PBS}$ and resuspended in $1 \times$ binding buffer. Approximately $10^{5}$ cells were incubated with $2 \mu \mathrm{l}$ of annexin V fluorescein isothiocyanate conjugate (FITC) and $1 \mu \mathrm{l}$ of propidium iodide (BD biosciences, US). The sperm were vortexed and incubated for $15 \mathrm{~min}$ at room temperature in the dark. After that $400 \mu \mathrm{l}$ of $1 \times$ binding buffer was added and the samples were analysed for early and late apoptosis by flow cytometry (Fach calibre, Becton Dickinson, USA).

\section{Detection of lipid peroxidation measurement by monitoring of MDA with high performance liquid chromatography (HPLC)}

Sperm samples were prepared with a modified technique from Tüközkan's method and acetonitrile (HPLC grade) was added to precipitate the proteins. The suspension was mixed for $30 \mathrm{~s}$ and centrifuged at $13000 \mathrm{~g}, 10 \mathrm{~min}$ at $4^{\circ} \mathrm{C}$. The filtered supernatant sample $(250 \mu \mathrm{l})$ was then mixed with $25 \mu \mathrm{l}$ dinitrophenylhydrazine solution (Sigma-Aldrich Corp., MI, USA), incubated for $10 \mathrm{~min}$ at room temperature and injected onto HPLC system. The MDA standard curve was prepared. The samples were analysed on SHIMADZU apparatus (Japan) with analytical column Prodigy ODS3 $5 \mu \mathrm{m} 100 \AA$, $250 \times 4.6 \mathrm{~mm}$ (Phenomenex, USA). Acetonitrile-ultra pure water $(38: 62, \mathrm{v} / \mathrm{v})$ containing $0.2 \%(\mathrm{v} / \mathrm{v})$ acetic acid was used in the mobile phase and UV detector was set at $310 \mathrm{~nm}$. MDA peaks were determined according to its retention time and confirmed by spiking with added exogenous standard. An aliquot of sample was allocated for the measurement of protein levels of tissue by Bradford method (Bio-Rad). Levels of MDA were calculated from a standard curve and expressed as $\mu \mathrm{M} / \mathrm{mg}$ protein.

\section{Superovulation and oocyte collection}

Three days before the experiment on IVF, female mice were superovulated by injection of pregnant mare serum gonadotropin (Sigma-Aldrich, USA) 5 IU to stimulate the oocyte growth. At 48-h interval, 5 IU of chorionic gonadotropin (Merck, USA) was injected to induce ovulation. Ten hours later, the cumulus-oocyte-complexes (COC) were collected from the oviduct and incubated in fertilization medium (Life Global group, USA) for 3-4 h for oocyte maturation until the time of insemination.

\section{In vitro fertilization and culture}

To study the effect of lycopene on oocyte and in vitro fertilization (IVF) outcome, oocytes were incubated with lycopene $5,10,25 \mu \mathrm{M}$ for $1 \mathrm{~h}$ before the insemination. Total $5 \times 10^{5}$ sperm were inseminated with 5-10 oocytes in a 4-well plate incubated with triple gas mixture of $6 \% \mathrm{CO}_{2}, 5 \% \mathrm{O}_{2}$ and $89 \% \mathrm{~N}_{2}$ 
at $37^{\circ} \mathrm{C}$. After $4 \mathrm{~h}$ of sperm and oocyte insemination, the COC were denuded to remove the remained cumulus cells and oocytes were transferred to culture in cleavage medium (Life Global group, USA) under oil. Cumulus cells and sperm collected from the inseminated wells were analysed for MDA levels. Fertilization was checked by presenting of 2 pronuclei at $16-19 \mathrm{~h}$ or 2-cell embryos at 34$36 \mathrm{~h}$ post-insemination under an inverted microscope (Olympus 1X71, USA). The experiments were performed in duplicate and at least in 2 independent experiments.

\section{Evaluation of embryo development and embryo grading}

The fertilization and cleavage rate of embryo development were recorded and embryo grading was examined by the number, size, shape of blastomeres and degree of fragmentation. The number of oocyte degeneration defined as dark, brown and clumping ooplasm was observed. The embryos are supposed to be 2 blastomeres on day 1, 4-8 blastomeres on day 2 , and become morula or early blastocyst on days $3-4$

The grading of embryo can be classified as follows. Grade 1 (good), less than 10\% fragmentation, stage-specific cell size, equal blastomeres; Grade 2 (fair), $10-25 \%$ fragmentation, stage-specific cell size for majority of cells and no evidence of multinucleation; Grade 3 (poor), larger than 25\% fragmentation, cell size not stage specific and evidence of multinucleation

\section{Statistic analysis}

Sperm motility, sperm apoptosis, and cleavage rate were presented as a percentage. Continuous and ordinal data were calculated as mean \pm SEM unless otherwise specified. Statistical analysis was performed using SPSS 17 for Windows (SPSS Inc., Chicago, IL, USA). Kruskal-Wallis nonparametric tests were used to analyse the difference between independent samples. A $p$-value of $<0.05$ was considered statistically significant.

\section{RESULTS}

The average concentration of pooled sperm was $26 \times 10^{5}$ cells $/ \mathrm{ml}$ with $60 \%$ of sperm motility at the beginning. After a 4-h incubation, sperm motility decreased in all groups compared to the untreated control. The treatment group of lycopene $10 \mu \mathrm{M}$ showed that sperm motility was comparable to the untreated control. However, treatment of lycopene with 3 concentrations $(5,10,25 \mu \mathrm{M})$ did not have

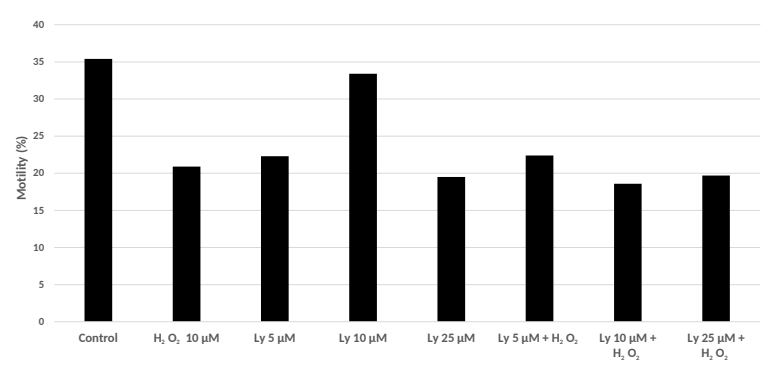

Fig. 1 The effect of lycopene with and without $\mathrm{H}_{2} \mathrm{O}_{2}$ induced oxidative stress on percentage of sperm motility after incubation for $4 \mathrm{~h}$. The mean of sperm motility was not significantly different $(p=0.36)$.

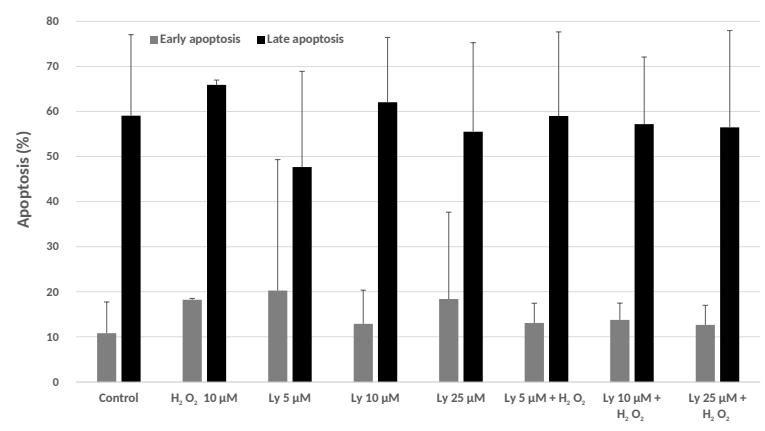

Fig. 2 The effect of lycopene on the percentage of early and late sperm apoptosis which was not significantly different among all groups $(p>0.05)$.

significant effect on protecting the sperm from the decline in motility (Fig. 1). Comparing between motility of the sperm treated with various concentrations of lycopene in $\mathrm{H}_{2} \mathrm{O}_{2}$ induced oxidative stress to the sperm treated with $\mathrm{H}_{2} \mathrm{O}_{2}$ group, there was no statistically difference in sperm motility.

The effect of lycopene on the percentage of early and late sperm apoptosis detected by flow cytometry showed that the percentage of early apoptosis was not significantly changed among all the groups. In the non-oxidative stress induced condition, there was a trend that lycopene may decrease the rate of late apoptosis in the group of lycopene 5 and $25 \mu \mathrm{M}$ compared to the untreated control. However, it did not reach statistically significance. Among the groups with $\mathrm{H}_{2} \mathrm{O}_{2}$ induced oxidative stress, the percentage of both early and late apoptosis was slightly decreased in all 3 groups of lycopene. However, it was not statistically significant (Fig. 2). The MDA levels of all treatment groups were slightly decreased compared to the control group but the decline levels were not statistically different.

The effect of lycopene on oocytes with IVF 


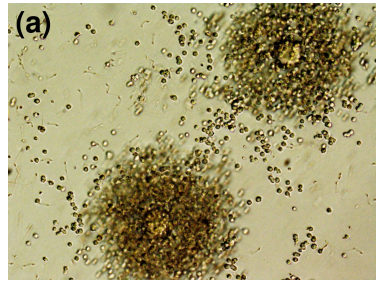

(c)
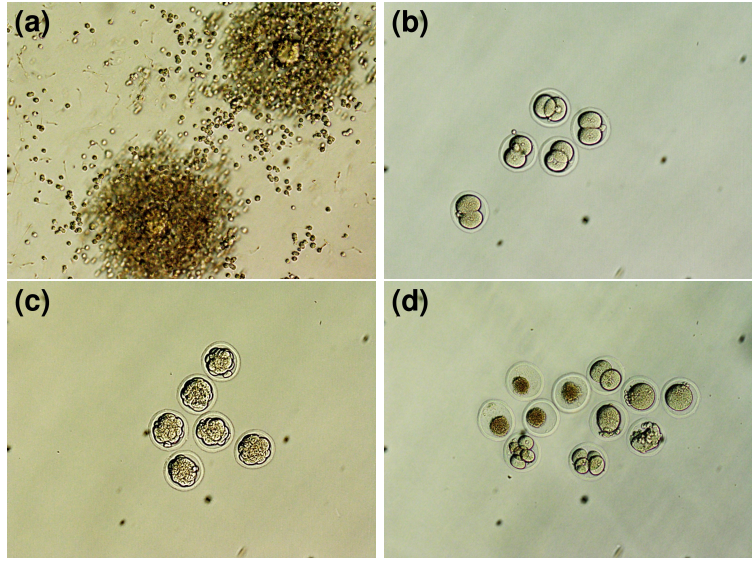

(d)

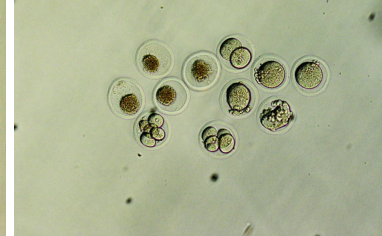

Fig. 3 In vitro fertilization process and embryo evaluation; (a) oocytes with cumulus-oophorus complex and sperm in the inseminated well, (b) two-cell embryos with good quality, (c) morula stage, (d) multiple stages of embryo with degenerated oocytes (dark, brown and clumping ooplasm).

Table 1 Cleavage rate, oocyte degeneration rate, and good-grade embryos in the lycopene-coincubated oocytes.

\begin{tabular}{lccc}
\hline $\begin{array}{l}\text { Group } \\
\text { (number of oocytes) }\end{array}$ & $\begin{array}{c}\text { Cleavage } \\
\text { rate }(\%)^{\mathrm{a}}\end{array}$ & $\begin{array}{c}\text { Degen. } \\
\text { rate }(\%)^{\mathrm{b}}\end{array}$ & $\begin{array}{c}\text { Good-grade } \\
\text { embryo }(\%)^{\mathrm{c}}\end{array}$ \\
\hline Control (30) & 50.0 & 16.7 & 66.7 \\
Lycopene 5 $\mu \mathrm{M}(49)$ & 49.0 & 14.3 & 62.5 \\
Lycopene 10 $\mu \mathrm{M}(57)$ & 36.8 & 26.3 & 57.1 \\
Lycopene 25 $\mu \mathrm{M}(40)$ & 17.5 & 62.5 & 71.4 \\
\hline
\end{tabular}

${ }^{\text {a }} p=0.97$,
${ }^{\text {b }} p=0.68$,
${ }^{\text {c }} p=0.94$.

outcome was evaluated for the control group and lycopene-coincubated oocytes at concentrations of 5, 10, $25 \mu \mathrm{M}$ (Fig. 3). The data showed that the higher lycopene concentration, the lower cleavage rate of embryos was observed. This decline in cleavage rate was not significantly different. The oocyte degeneration rate seemed to be quite high in the group of oocyte coincubated with lycopene $25 \mu \mathrm{M}(62 \%)$. When evaluated the embryo grading among cleaved embryos, it was shown that the percentage of good-grade embryo was comparable among various lycopene concentrations (Table 1). The MDA levels of cumulus cells and sperm in each inseminated well were the highest in the group of oocyte coincubated with lycopene $25 \mu \mathrm{M}$ but the levels were not significantly different (Fig. 4). This highest levels of MDA may be associated with the percentage of oocyte degeneration.

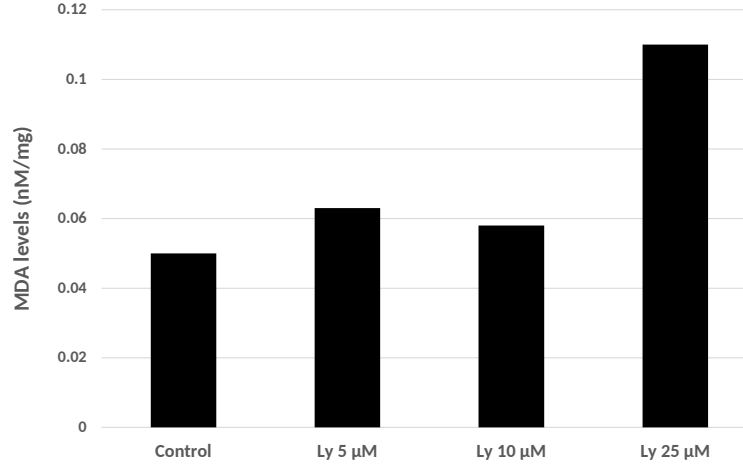

Fig. 4 The MDA levels from the inseminated well of the lycopene-treated oocytes in IVF slightly increased with the lycopene concentration compared to the control which was correlated with the percentage of oocyte degeneration $(p>0.05)$.

\section{DISCUSSION}

Lycopene has potent antioxidative properties and has shown beneficial effects in many health conditions related to oxidative stress. Lycopene is a carotenoid but lacks provitamin A properties as it contains acyclic end groups. Unlike other vitamin A derivatives, lycopene has no teratogenic effects. Hence lycopene can be consumed by reproductive persons and used in the study of the reproductive system. In the process of assisted reproductive technology to treat infertility, several factors can induce ROS generation such as sperm damage during sperm preparation by high speed centrifugation, dead sperm and degenerated cumulus cells, high oxygen concentration in the culture media and system, and exposure to extreme temperatures or visible light. The authors hypothesized that adding lycopene in the culture media for sperm and oocyte might improve sperm parameters and embryo developmental rate. According to literature review, there is little evidence about using lycopene in the IVF system.

After incubating sperm in lycopene 5, 10, $25 \mu \mathrm{M}$, the sperm motility was decreased in both normal condition and $\mathrm{H}_{2} \mathrm{O}_{2}$ induced oxidative stress condition. This result was quite similar to the previous study by Zini et al that preincubation of sperm with lycopene 2 and $5 \mu \mathrm{M}$ for $30 \mathrm{~min}$ at $25^{\circ} \mathrm{C}$ did not protect sperm from the decline in motility. Our results confirm that even lycopene concentration higher than $5 \mu \mathrm{M}$ with longer time of incubation had no protective effect on sperm motility. However, in the normal condition sperm treated with lycopene $10 \mu \mathrm{M}$ showed the highest motility rate compared to 
lycopene 5 and $25 \mu \mathrm{M}$. This may be interpreted that the protective effect of lycopene on sperm motility was not a dose dependent manner. This hypothesis may be supported by the study of Akalin et al about the effect of lycopene on the ram sperm motility during liquid storage at $5{ }^{\circ} \mathrm{C}^{31}$. Supplementation of lycopene at $0.5 \mathrm{mM}$ dose for 24,48 , and $72 \mathrm{~h}$ resulted in higher sperm motility rate compared to 1 and $2 \mathrm{mM}$ lycopene. The results by Zini et al revealed that preincubation lycopene $5 \mu \mathrm{M}$ for $30 \mathrm{~min}$ had a protective effect against oxidative sperm DNA damage even though the motility was not improved ${ }^{30}$. The proposed mechanism was that lycopene may reduce lipid peroxidation at the sperm plasma membrane and prevent oxidative DNA injury. In an environment of oxidative stress, sperm apoptosis may have an effect on sperm DNA damage and impair fertilization leading to poor quality embryo.

In our study, the percentage of early apoptotic rate remained the same in all studied groups. On the contrary, the percentage of late apoptosis in the normal condition had a trend to decrease slightly in the group of lycopene 5 and $25 \mu \mathrm{M}$. In the $\mathrm{H}_{2} \mathrm{O}_{2}$ induced oxidative stress condition, the percentage of late apoptosis was decreased in all groups of sperm treated with lycopene. This result could be explained as that lycopene may prevent further sperm damage from early to late apoptosis; but whether lycopene can reverse sperm apoptosis is still questionable. Probably, the process of sperm apoptosis is irreversible. In this study, the MDA levels which indicate oxidative stress showed that lycopene treated groups had lower MDA levels even though it was not statistically significant. The decreased MDA levels and late apoptotic rate could represent an antioxidative property of lycopene but the in vitro effect of lycopene may be mild. In a vivo study, Wistar rats received lycopene gavage at the dose of $4 \mathrm{mg} \mathrm{kg}^{-1} \mathrm{day}^{-1}$ and later were treated with lipopolysaccharide via intraperitoneal injection to induce lipid peroxidation, lycopene was found to exhibit a protective effect on testicular mitochondria damage and increase sperm count and motility ${ }^{32}$. Most of the scientific data about antioxidative effect of lycopene on anticancer in prostate gland have been conducted with lycopene enriched-food consumption such as tomatoes. Supplementation of other antioxidants in the culture system was studied in mouse by Donnelly et al. The study showed that supplementation of vitamin $C$ and $E$ in sperm preparation could reduce ROS levels but sperm motility was not improved ${ }^{33}$. Thus adding any antioxidant to the culture medium may not affect the sperm parameters.

The data of antioxidative effect of lycopene on female gametes are scarce. From an intensive literature review, only one published paper about female gamete and lycopene effect was found. Chinese hamster ovary cells were treated with hydrogen peroxide and with various concentrations of lycopene $(10,25,50 \mu \mathrm{M})$, simultaneously or post treatment. The results showed that these three concentrations of lycopene reduced the DNA damage, especially in the group with simultaneous treatment. From this study, lycopene may have chemopreventive activity on oxidative stress in Chinese hamster ovary cell ${ }^{34}$.

In our study, cumulus-oophorus complex were incubated with lycopene supplementation in the culture medium. Interestingly, rate of oocyte degeneration was the highest when the oocytes were incubated with lycopene $25 \mu \mathrm{M}$ and the cleavage rate in this group was also decreased. This result was correlated with the MDA levels and may be interpreted as that high concentration of lycopene may have a negative effect on oocyte function and embryo cleavage. When the cleaved embryo quality was analysed, the percentage of good-grade embryos was not different. However, in the IVF unit the number of cleaved embryo is very important to increase the chance of achieving pregnancy.

In this study, the interim analysis was performed because preliminary data showed that lycopene may not improve sperm motility and not significantly reduce early and late apoptosis. Furthermore, lycopene may also have detrimental effect on oocytes in the culture system. Due to animal ethics concerns, further experiment could not be continued. Although lycopene has a very potent antioxidative effects, it has a limitation because it is expensive and unstable. Synthetic lycopene should be kept under inert gas in lightproof containers and stored in a cool place to prevent isomerization and oxidation, making lycopene difficult to use in a culture system ${ }^{21}$.

In conclusion, administration of lycopene 5,10 , $25 \mu \mathrm{M}$ in sperm and oocyte culture media may have little antioxidative effects in in vitro fertilization system. The optimal concentration of antioxidant is also challenging and problematic because small amounts of ROS are crucial in normal physiology. It should be kept in mind that antioxidant therapy may lead to negative effects if a certain threshold concentration is exceeded. Thus supplementation of lycopene in the culture media of IVF system should be further studied to determine the most effective dose and incubation time. 
Acknowledgements: This study was supported by endowment fund for research, Naresuan University, Thailand. The authors would like to acknowledge Mr Sang Sriumpai for assisting in flow cytometry analysis.

\section{REFERENCES}

1. Agarwal A, Saleh RA, Bedaiwy MA (2003) Role of reactive oxygen species in the pathophysiology of human reproduction. Fertil Steril 79, 829-43.

2. Agarwal A, Gupta S, Sharma RK (2005) Role of oxidative stress in female reproduction. Reprod Biol Endocrinol 3, 28-49.

3. Lavranos G, Balla M, Tzortzopoulou A, Syriou V, Angelopoulou R (2012) Investigating ROS sources in male infertility: a common end for numerous pathways. Reprod Toxicol 34, 298-307.

4. Guerin P, El Mouatassim S, Menezo Y (2001) Oxidative stress and protection against reactive oxygen species in the pre-implantation embryo and its surroundings. Hum Reprod Update 7, 175-89.

5. Halliwell B, Gutteridge JM (1984) Lipid peroxidation, oxygen radicals, cell damage, and antioxidant therapy. Lancet 323, 1396-7.

6. Valko M, Leibfritz D, Moncol J, Cronin MT, Mazur M, Telser J (2007) Free radicals and antioxidants in normal physiological functions and human disease. Int J Biochem Cell Biol 39, 44-84.

7. Gomez E, Buckingham DW, Brindle J, Lanzafame FM, Irvine DS, Aitken RJ (1996) Development of an image analysis system to monitor the retention of residual cytoplasm by human spermatozoa: correlation with biochemical markers of the cytoplasmic space, oxidative stress, and sperm function. $J$ Androl 17, 276-87.

8. Aitken RJ, Lambourne S, Gibb Z (2014) The John Hughes memorial lecture: aspects of sperm physiology-oxidative stress and the functionality of stallion spermatozoa. $J$ Equine Vet Sci 34, 17-27.

9. Aprioku JS (2013) Pharmacology of free radicals and the impact of reactive oxygen species on the testis. J Reprod Infertil 14, 158-72.

10. Lanzafame FM, La Vignera S, Vicari E, Calogero AE (2009) Oxidative stress and medical antioxidant treatment in male infertility. Reprod Biomed Online 19, 638-59.

11. Si Y, Okuno M (1999) Role of tyrosine phosphorylation of flagellar proteins in hamster sperm hyperactivation. Biol Reprod 61, 240-6.

12. Combelles CM, Gupta S, Agarwal A (2009) Could oxidative stress influence the in-vitro maturation of oocytes? Reprod Biomed Online 18, 864-80.

13. Agarwal A, Gupta S, Sharma R (2005) Oxidative stress and its implications in female infertility - clinician's perspective. Reprod Biomed Online 11, 641-50.

14. Agarwal A, Gupta S, Sikka S (2006) The role of free radicals and antioxidants in reproduction. Curr Opin Obstet Gynecol 18, 325-32.

15. Bedaiwy M, Agarwal A, Said TM, Goldberg JM, Sharma RK, Worley S, Falcone T (2006) Role of total antioxidant capacity in the differential growth of human embryos in vitro. Fertil Steril 86, 304-9.

16. Goud AP, Goud PT, Diamond MP, Gonik B, AbuSoud HM (2008) Reactive oxygen species and oocyte aging: role of superoxide, hydrogen peroxide, and hypochlorous acid. Free Radic Biol Med 44, 1295-304.

17. Burton GJ, Jauniaux E (2011) Oxidative stress. In: Brosens I (ed) Placental Bed \& Maternal-Fetal Disorders, Best Practice \& Research Clinical Obstetrics \& Gynaecology vol 25, 287-99.

18. Ratnam DV, Ankola DD, Bhardwaj V, Sahana DK, Kumar MN (2006) Role of antioxidants in prophylaxis and therapy: A pharmaceutical perspective. $J$ Contr Release 113, 189-207.

19. Stahl W, Sies H (2005) Bioactivity and protective effects of natural carotenoids. Biochim Biophys Acta 1740, 101-7.

20. Rao AV, Rao LG (2007) Carotenoids and human health. Pharmacol Res 55, 207-16.

21. Roldán-Gutiérrez JM, Luque de Castro MD (2007) Lycopene: the need for better methods for characterization and determination. Trends Anal Chem 26, 163-70.

22. Breeman R, Pajkovic N (2008) Multitargeted therapy of cancer by lycopene. Canc Lett 269, 339-51.

23. Ilic D, Misso M (2012) Lycopene for the prevention and treatment of benign prostatic hyperplasia and prostate cancer: a systematic review. Maturitas 72, 269-76.

24. Lee LK, Foo KY (2013) An appraisal of the therapeutic value of lycopene for the chemoprevention of prostate cancer: A nutrigenomic approach. Food Res Int 54, 1217-28.

25. Srinivasan M, Sudheer AR, Pillai KR, Kumar PR, Sudhakaran PR, Menon VP (2007) Lycopene as a natural protector against gamma-radiation induced DNA damage, lipid peroxidation and antioxidant status in primary culture of isolated rat hepatocytes in vitro. Biochim Biophys Acta 1770, 659-65.

26. Tüközkan N, Erdamar H, Seven I (2006) Measurement of total malondialdehyde in plasma and tissues by high-performance liquid chromatography and thiobarbituric acid assay. Furat Med J 11, 88-92.

27. McClain RM, Bausch J (2003) Summary of safety studies conducted with synthetic lycopene. Regul Toxicol Pharmacol 37, 274-85.

28. Henkel RR, Schill WB (2003) Sperm preparation for ART. Reprod Biol Endocrinol 1, 108-30.

29. Agarwal A, Said TM, Bedaiwy MA, Banerjee J, Alvarez JG (2006) Oxidative stress in an assisted reproductive techniques setting. Fertil Steril 86, 503-12.

30. Zini A, Gabriel MS, Libman J (2010) Lycopene sup- 
plementation in vitro can protect human sperm deoxyribonucleic acid from oxidative damage. Fertil Steril 94, 1033-6.

31. Akalin PP, Bucak MN, Güngör Ş, Başpinar N, Çoyan K, Dursun Ş, İli P, Aksoy A, et al (2016) Influence of lycopene and cysteamine on sperm and oxidative stress parameters during liquid storage of ram semen at $5^{\circ} \mathrm{C}$. Small Rumin Res 137, 117-23.

32. Aly HA, El-Beshbishy HA, Banjar ZM (2012) Mitochondrial dysfunction induced impairment of spermatogenesis in LPS-treated rats: modulatory role of lycopene. Eur J Pharmacol 677, 31-8.

33. Donnelly ET, McClure N, Lewis SE (1999) Antioxidant supplementation in vitro does not improve human sperm motility. Fertil Steril 72, 484-95.

34. Scoloastici C, Alves de Lima RO, Barbisan LF, Ferreira AL, Ribeiro DA, Salvadori DMF (2007) Lycopene activity against chemically induced DNA damage in Chinese hamster ovary cells. Toxicol In Vitro 21, 840-5. 